\title{
Graves' Disease and Marine Lenhart Syndrome: A Rare Clinical Presentation
}

\author{
Fernanda Cardia Martins Ribeiro', Larissa Alves Ruas', Aluysio Leal Júnior'1, \\ Adriana Andrade de Sousa1, Adriana Farias Araújo', Jonathan Mwambire², \\ Ana Luísa Conceição de Jesus ${ }^{3}$, Renata Maksoud Bussuan'1, Larissa Bianca Cunha de Sá1, \\ Alberto Krayyem Arbex ${ }^{1,4}$
}

\author{
${ }^{1}$ IPEMED Medical School/AFYA Educational, Postgraduation in Endocrinology, Rio de Janeiro, Brazil \\ ${ }^{2}$ Internal Medicine, Malteser Krankenhaus Franziskus Hospital, Flensburg, Germany \\ ${ }^{3}$ Department of Pediatrics, University of São Paulo (USP) Medical School, São Paulo, Brazil \\ ${ }^{4}$ Medical Clinic in Endocrinology and Diabetology, Schleswig-Flensburg, Germany \\ Email: ribeiro.fernanda@globo.com
}

How to cite this paper: Ribeiro, F.C.M., Ruas, L.A., Júnior, A.L., de Sousa, A.A., Araújo, A.F., Mwambire, J., de Jesus, A.L.C., Bussuan, R.M., de Sá, L.B.C., Arbex, A.K. (2019) Graves' Disease and Marine Lenhart Syndrome: A Rare Clinical Presentation. Health, 11, 1169-1176.

https://doi.org/10.4236/health.2019.119091

Received: August 23, 2019

Accepted: September 24, 2019

Published: September 27, 2019

Copyright $\odot 2019$ by author(s) and Scientific Research Publishing Inc. This work is licensed under the Creative Commons Attribution International License (CC BY 4.0).

http://creativecommons.org/licenses/by/4.0/

\begin{abstract}
The aim of this study is to review and discuss the clinical features and treatment options for the Marine-Lenhart syndrome, an uncommon thyroid disturb. The methodology adopted was a bibliographic research. The results of this study show that different mechanisms are implicated in the pathogenesis of Graves' disease and in the nodular formation of thyroid tissue with functional autonomy. Graves' disease is caused by an autoimmune process that involves the entire thyroid gland and is characterized by the presence of TSH receptor stimulating antibodies. The present study concludes that caution should be exercised in interpreting thyroid disease in Graves' disease. The treatment of thyrotoxicosis requires high doses of oral drugs. Relapsing antithyroid therapy occurs soon after oral antithyroid therapy is discontinued. This presentation should alert the physician about the existence of toxic nodules, and about the possibility of a Marine-Lenhart Syndrome.
\end{abstract}

\section{Keywords}

Graves' Disease, Marine-Lenhart Syndrome, Thyroid Gland, Thyroid Nodules

\section{Introduction}

Marine-Lenhart syndrome describes the association between Graves' disease and autonomously functioning thyroid nodules (AFTN), such as toxic adenoma or toxic multinodular goiter. The two diseases may coexist or may be present at 
different times in the same patient [1].

Following a hyperthyroidism of Graves' disease with strongly positive antithyroid antibodies treated with radioiodine therapy, the patient may develop a bifocal autonomous nodule. This phenomenon is known as Marine-Lenhart syndrome. In this particular case, it appears that autonomous nodules are a consequence of radioactive iodine treatment of Graves' disease [2].

Thyroid nodules in patients with Graves' disease (GD) are common, and the incidence of coexisting thyroid carcinoma is a hotly debated issue, which is addressed in this study. Thyrotoxicosis results from autonomic thyroid hyperfunction or destructive processes. A representative autonomy of the thyroid (hyperthyroidism) is Graves' disease, a common autoimmune disease caused by TSH receptor stimulating antibodies (TRAb). Hyperthyroidism also results from autonomic adenomas (solitary toxic adenoma or Plummer's disease) [3].

Marine-Lenhart syndrome is a rare disease consisting of both Graves' disease and Plummer's disease. There have been several case reports describing Marine-Lenhart syndrome; however, to the best of our knowledge, all previously reported cases except two involved a positive TRAbtitres [2].

In this study, the objective is to review and discuss the clinical features and treatment options for Marine-Lenhart syndrome. This is a rare clinical condition that deserves further discussion for a better understanding of the disease.

\section{Methodology}

For the development of this study, a descriptive method was adopted, for the analysis of the current medical literature, with a qualitative approach. The study was conducted through a thorough literature review research, since there are few cases reported. For Lakatos and Marconi (2007), this type of research is defined as the survey, selection and documentation of all bibliography that has been published on the subject, and enables the researcher to contact these materials and deepen their knowledge on the subject. The search was performed with the databases of Latin American and Caribbean Health Sciences Literature (LILACS), Scientific Electronic Library Online (SCIELO), monographs, dissertations and scientific articles, without a date limitation.

The inclusion criteria of the studies for the bibliographic survey were full texts in Portuguese, with unpaid and free access in the databases mentioned above. Exclusion criteria were studies that did not meet the study objectives. Articles were searched in English, Portuguese and Spanish.

The database search was conducted in February 2018. The key terms used for the research were Graves' Disease, Marine-Lenhart Syndrome and Treatment.

\section{Background}

Graves' disease is an autoimmune disease in which stimulatory autoantibodies bind to the TSH receptor and activate glandular function, leading to hyperthyroidism. 25\% - 30\% of Graves' disease patients are reported to harbor thyroid 
nodules. Mostly, these nodules are cold, benign and multiple, but $1 \%-2.5 \%$ are associated with hot autonomous nodules [4].

GD is an autoimmune disorder in which TSH receptors (TSH) stimulate thyroid stimulating hormone (TSH), increasing the production and release of thyroid hormones. In patients with this disorder, immunological examination commonly shows a transient increase in TRAb and thyroid peroxidase antibodies (TPO Abs) that are undetectable in toxic adenoma [5].

Graves' disease is believed to be caused by an autoimmune process in which the stimulatory autoantibodies bound to the TSH receptor and activates glandular function, leading to hyperthyroidism. Thyroid nodules accompany Graves' Disease at rates ranging from $25 \%$ to $30 \%$ of patients [1].

TSH plays a central role in thyroid growth and normal functioning and appears to play a similar role in the growth and development of thyroid cancer. The close relationship between TSH and TSH-R-stimulating antibodies (TSH-R $\mathrm{AB})$ seen in Graves' disease led to the realization that thyroid cancer occurring in the setting of this disease may become more aggressive as a result of stimulation by these autoantibodies [6].

Several conditions, including parathyroidectomy, acute or subacute thyroiditis, and percutaneous injection of ethanol, have been reported to damage follicular cells, and the TSH receptor antigen released from damaged cells may trigger the autoimmune response that resulted in GD. With this similar mechanism, radioiodine therapy for autonomous thyroid disease can induce GD with temporary TRAb increase at a very low rate of $1.3 \%$ [7].

Wanderley (2015, p. 4) reports that: “Graves' Disease is the most common form of hyperthyroidism in sufficient iodine areas $(60 \%-80 \%)$. It is an autoimmune disease characterized by the presence of thyrotoxicosis, diffuse toxic goiter, ophthalmopathy, and occasionally infiltrative dermopathy (pretibial myxedema). In turn, papillary carcinoma is the most frequent thyroid tumor and is responsible for $80 \%$ of thyroid cancer cases in the USA" [8].

A high percentage of these nodules are hypoactive according to thyroid exams (over 95\%) and a small percentage of these hyperactive nodule subjects. Thus, these subjects have thyrotoxicosis secondary to Graves' disease and toxic nodular goiter. These occasional patients are called Marine-Lenhart syndrome. A case of Marine-Lenhart Syndrome has been presented and the therapeutic approach of these subjects has been discussed [9].

Marine-Lenhart syndrome is a variant of Graves' disease, with accidentally functioning nodules that respond to thyroid stimulating hormone but do not respond to thyroid stimulating immunoglobulins. Marine-Lenhart syndrome, also known as nodular Graves' disease, is the coincidence of Graves' disease with TSH-sensitive functioning nodules. The syndrome was initially described in 1911 by Marine and Lenhart and is now considered a distinct sub-entity of Graves' disease [4].

In 1911 Marine and Lenhart published their observations on histopathology and iodine content of the thyroid gland in exophthalmic goiter (Graves' disease). 
Eight patients with follicular adenoma were included in this study. Subsequently, Marine described three more cases, making a total of 11 cases. In five of the eleven patients, the iodine concentration in the nodule was $0 \%-3 \%$ of extranodular tissue, but in six of the patients it ranged from $12 \%$ to $40 \%$ [10]. After its description by Marine and Lenhart, exophthalmic goiter syndrome with incident functional nodule(s) was accepted as a distinct subdivision of Graves' disease [10].

Giuffridareports that Graves' disease and autonomic function thyroid nodules (NFAT) cause thyrotoxicosis by different pathophysiological mechanisms. The coexistence of both diseases was called "Marine-Lenhart syndrome". Since the first description in 1911 by Marine and Lenhart [11], the presence of focal autonomy in patients with Graves' disease has been reported by numerous authors with different presentations, with a general prevalence of such an association of $2.7 \%$ to $4.1 \%[12]$.

Marine-Lenhart syndrome has been described as a variant of Graves' disease with the following criteria: 1) Thyroid CT scan shows enlarged gland and 1 or 2 malfunctioning nodules; 2) The nodule is dependent on TSH and the paranodular tissue is independent of TSH; 3) After endogenous or exogenous TSH stimulation, a return of nodule function may be demonstrated; and 4) The nodule is histologically benign [2]. It is reported to be quite rare with a prevalence of $2.7 \%-4.1 \%$ in patients with Graves' disease [9].

Thyroid scintigraphy shows the typical finding of increased activity with decreased fundus, but with one or more cold nodules (suppressed by TSH). After therapy with antithyroid drugs or radioactivity of I-131, the nodules could accumulate radiotracer and appear as hot nodules as the TSH level begins to rise [13].

In recent years, several articles have been published on the development of Graves' disease shortly after radioactive iodine therapy for AFTNs. The incidence of this event is significantly higher, especially in patients with early high serum thyroid peroxidase (TPO-Ab) antibodies as well as in early negative TPOAb patients who became positive for TPO-Ab after treatment. The risk of thyroid malignancy in patients with concomitant autonomous functioning nodules remains underestimated in clinical practice due to its low occurrence and the misconception that the coexistence of a "hot" nodule and thyroid carcinoma is very rare [14].

The probability of a carcinoma within a warm lump is less than $1 \%$ [13]. Therefore, it is commonly believed that the diagnosis of AFTN can almost always rule out nodule malignancy. According to the guidelines, nodules that are hot on scintigraphy should be excluded from FNAB [15].

The type of scintigraphy employed in this type of image is important. Thyroid tomography may be performed with iodine-123, iodine-131 or technetium-99 labeled pertechnetate. Iodine isotopes, which are both entrapped and organically attached to the thyroid, are preferred, as $3 \%-8 \%$ of the nodules that appear to function in pertechnetate scanning may appear to be non-functioning in radioi- 
odine (RAI) scanning, some of which may to be malignant [15].

\section{Clinical and Laboratory Manifestations of the Syndrome}

Marine-Lenhart syndrome is defined as the co-occurrence of Graves' disease and functional nodules. The vast majority of autonomic adenomas are benign and functional thyroid carcinomas are considered rare [6].

Well-differentiated papillary carcinomas are found to coexist with surgically treated GDs (7.8\%) and occur most frequently in GDs with palpable nodular lesions (35\%). Although most (88\%) of coexisting carcinomas are microcarcinomas, the presence of palpable nodules justifies further evaluation and follow-up [16].

The BRAF V600E mutation is generally associated with increased malignancy and aggressiveness in papillary thyroid carcinomas and in most cases with typical thyroid malignancy ultrasound features [6].

The coexistence of hyperthyroidism and thyroid carcinoma is no longer considered a rare finding, but there is no consensus yet on many aspects of this clinical condition [17].

The incidence of these nodules reported in the literature ranges from $12.8 \%$ to $33.6 \%$. The main factor influencing the difference in reported prevalence of these nodules is the method used to detect them (eg, clinical, thyroid, ultrasound, or combined). When only clinical examination and tomography are used, the prevalence may be much lower than when compared to ultrasound. In a multicenter study of 140 cases out of 557, reported cases that had only 40 nodules $(28.6 \%)$ which were palpable cold nodule and scintigraphy showed in 54 (38.6\%) cases only. However, ultrasound could detect 116 (82.9\%) of these cases (prevalence of 25.1\%) [18].

The prevalence of concomitant thyroid carcinoma with Graves' disease was reported to range from $1.1 \%$ to $7.1 \%$. Most of these carcinomas are due to cold nodules. Thyroid Papillary Microcarcinoma is the most common histological subtype [19].

According to Bartalena (2009), RAI treatment has a risk of progressing to Graves' ophthalmopathy. In addition, it has been suggested that functioning nodules in patients with Marine-Lenhart syndrome are more resistant to radius compared to Graves' disease without nodules [20].

Thus, the ARF treatment was discarded in our patient based on these reports. The patient underwent thyroidectomy for hyperthyroidism and a hyperfunctioning nodule. There is only one case in the literature reporting micropapillary thyroid carcinoma detected in the hyperfunctioning nodule in a patient with Graves' disease [21].

Carcinomas reported within hyperfunctioning thyroid nodules are most often papillary microcarcinomas. Malignant tumors larger than $1 \mathrm{~cm}$ in diameter are very rare [14].

Currently, there is no consensus on the proper work of these patients. Ultra- 
sound may be the most sensitive method for detecting thyroid nodules. But the clinical significance of most of these nodules remains controversial, as thyroid nodules are often found in thyroid ultrasound studies. FNAC is an excellent tool in the evaluation of the thyroid nodule. But it is difficult to perform in small nodules. Because of this and the problem of bleeding, interpretation of the results may not be easy in the presence of hyperplastic tissue in Graves' disease. This is particularly relevant in cases that the nodule was developed after treatment with radioactive iodine [22].

Although most incidentally discovered nodules cannot be of any clinical significance, many believe that in the presence of a palpable cold nodule in the background of Graves' disease the incidence of malignancy is high, and may range from $19 \%$ to $45.8 \%$ [23].

Some patients with toxic multinodular goiter may be tested positive for thyroid autoantibodies. The differential diagnosis between these patients and Marine-Lenhart syndrome is based on the appearance of thyroid tomography. In patients with toxic multinodular goiter, increased uptake is seen in areas of the nodules defined by ultrasound and the rest of the gland is suppressed.

In Marine-Lenhart syndrome, there is a diffuse and increased uptake in the gland in general, with nodule-enhanced uptake foci [23]. Most reports have shown extra nodular parenchymal hyperactivity and relative nodular hypoactivity on initial Marine-Lenhart syndrome scintigraphy [24]. It has been suggested that tissue within the nodule is suppressed by hyperactivity of the unaffected gland and therefore does not occupy tracer or RAI. However, increased homogeneous absorption was also observed in a report on extra nodular tissue with intense focal uptake in the nodules [9].

In addition, there is also a report showing similar activity between nodules and extranodular tissues. In this patient, diffusely homogeneous uptake throughout the gland and additional focal uptake with centrally hypoactive foci corresponding to the sonographically detected nodule were observed [25].

Total complement thyroidectomy after subtotal thyroidectomy is fraught with danger. The reported incidence of recurrent nerve palsy and hypothyroidism is often higher on reoperation compared with the primary operation. It can be argued that subtotal thyroidectomy is sufficient in these cases, since most of them are micro-carcinoma [26].

\section{Conclusions}

The purpose of this paper was to review and discuss the clinical features and treatment options for the Marine-Lenhart syndrome. The reports on this study show that different mechanisms are implicated in the pathogenesis of Graves' disease and in the nodular formation of thyroid tissue with functional autonomy.

Graves' disease has been found to be caused by an autoimmune process involving the entire thyroid gland and is characterized by the presence of TSH re- 
ceptor stimulating antibodies.

In conclusion, this paper points out that caution should be exercised in interpreting thyroid disease in Graves' disease. The treatment of thyrotoxicosis requires high doses of oral, or relapsing antithyroid therapy occurs soon after oral antithyroid therapy is discontinued. This should alert the doctor about the existence of toxic nodules, thus Marine-Lenhart Syndrome. More research on this topic is needed to further understand the mechanisms involved in this clinical entity.

\section{Acknowledgements}

The research team would like to thank AFYA Educational for supporting scientific publishing and for always searching international achievements within the global science community.

\section{Conflicts of Interest}

The authors declare no conflicts of interest regarding the publication of this paper.

\section{References}

[1] Giuffrida, G., et al. (2014) An Uncommon Case of Marine-Lenhart Syndrome. Arquivos Brasileiros de Endocrinologia \& Metabologia, Sao Paulo, 58, 398-401. https://doi.org/10.1590/0004-2730000003173

[2] Biersack, H.J. and Biermann, K. (2011) The Marine-Lenhart Syndrome Revisited. Wiener Klinische Wochenschrift, 123, 459-462. https://doi.org/10.1007/s00508-011-0029-5

[3] Franklyn, J.A. and Boelaert, K. (2012) Thyrotoxicosis. The Lancet, 379, 1155-1166. https://doi.org/10.1016/S0140-6736(11)60782-4

[4] Mohan, J.R., et al. (2013) Pertechnetate Thyroid Scan in Marine-Lenhart Syndrome. Indian Journal of Nuclear Medicine, 28, 125-126. https://doi.org/10.4103/0972-3919.118261

[5] Shen, G., et al. (2017) Serious Disease Following Radioiodine Therapy for Toxic Adenoma. Medicine (Baltimore), 96, e8550. https://doi.org/10.1097/MD.0000000000008550

[6] Scherer, T., et al. (2013) A Case of Simultaneous Occurrence of Marine-Lenhart Syndrome and a Papillary Thyroid Microcarcinoma. BMC Endocrine Disorders, 13, Article No. 16. https://doi.org/10.1186/1472-6823-13-16

[7] Nishikawa, M., et al. (1997) Coexistence of an Autonomously Functioning Thyroid Nodule in a Patient with Graves' Disease: An Unusual Presentation of MarineLenhart Syndrome. Endocrine Journal, 44, 571-574. https://doi.org/10.1507/endocrj.44.571

[8] Wanderley, B.R., et al. (2015) Traps in the Papuliferous Carcinoma Diagnoses Associated with the Graves' Disease. Re vista da Sociedade Brasileira de Clinica Medica, 13, 194-197.

[9] Cakir, M. (2005) Marine-Lenhart Syndrome. Journal of the National Medical Association, 97, 1036-1038. https://doi.org/10.1089/1050725041516995

[10] Harisankar, C.N., et al. (2013) Hybrid SPECT/CT Evaluation of Marine-Lenhart 
Syndrome. Clinical Nuclear Medicine, 38, e89-e90.

[11] Marine, D. and Lenhart, C.H. (1911) Pathological Anatomy of Exopthalmic Goiter. Archives of Internal Medicine, 3, 265-316. https://doi.org/10.1001/archinte.1911.00060090002001

[12] Charkes, N.D. (1972) Serious Disease with Functioning Nodules (Marine-Lenhart Syndrome). Journal of Nuclear Medicine, 13, 885-892.

[13] Graf, H. (2004) Nodular Thyroid Disease. Arquivos Brasileiros de Endocrinologia \& Metabologia, Sao Paulo, 48, 93-104. https://doi.org/10.1590/S0004-27302004000100011

[14] Polyzos, S.A. and Goulis, D.G. (2011) Coincidental Thyroid Papillary Microcarcinoma in a Patient Treated for a Toxic Adenoma of the Thyroid. Archives of Iranian Medicine, 14, 149-151.

[15] Atmaca, H., et al. (2015) Marine-Lenhart Syndrome with Papillary Thyroid Carcinoma. Journal of Research in Medical Sciences, 20, 412-415.

[16] Gerenova, J., et al. (2003) Prevalence of Thyroid Cancer in Graves' Disease: A Retrospective Study of a Cohort of 103 Patients Treated Surgically. European Journal of Internal Medicine, 14, 321-325. https://doi.org/10.1016/S0953-6205(03)00105-5

[17] Filho, A., Furtado, V.J., et al. (2001) Papillary Thyroid Carcinoma in Severe Disease. Revista do Colégio Brasileiro de Cirurgióes, Rio de Janeiro, 28, 245-248. https://doi.org/10.1590/S0100-69912001000400002

[18] Kraimps, J.L., et al. (2000) Multicentre Study of Thyroid Nodules in Patients with Graves' Disease. British Journal of Surgery, 87, 1111-1113.

[19] Majima, T., et al. (2005) Anaplastic Thyroid Carcinoma Associated with Graves' disease. Endocrine Journal, 52, 551-557. https://doi.org/10.1507/endocrj.52.551

[20] Bartalena, L. (2012) Prevention of Graves' Ophthalmopathy. Best Practice and Research in Clinical Endocrinology and Metabolism, 26, 371-379. https://doi.org/10.1016/j.beem.2011.09.004

[21] Hernán-Martínez, J., et al. (2010) Hyperthyroidism, Thyroid Nodule Hyperfunctioning, and Thyroid Cancer in a Young Female: A Rare and Unusual Coexistence. Puerto Rico Health Sciences Journal, 29, 78-82.

[22] Carnell, N.E. and Valentine, W.A. (1998) Thyroid Nodules in Graves' Disease: Classification, Characterization, and Response to Treatment. Thyroid, 8, 647-652. https://doi.org/10.1089/thy.1998.8.647

[23] Centeno, B.A., et al. (1996) Fine Needle Aspiration Biopsy of Thyroid Gland in Patients with Prior Graves' disease Treated with Radioactive Iodine. Morphologic Findings and Potential Pitfalls. Acta Cytologica, 40, 1189-1197. https://doi.org/10.1159/000333979

[24] Brahma, A., et al. (2012) The Oldest Case of Marine-Lenhart Syndrome? JRSM Short Reports, 3, 21. https://doi.org/10.1258/shorts.2011.011164

[25] Chatzopoulos, D., et al. (2007) Marine-Lenhart Syndrome and Radioiodine-131 Treatment. Thyroid, 17, 373-374. https://doi.org/10.1089/thy.2006.0150

[26] Mishra, A. and Mishra, S.K. (2001) Thyroid Nodules in Graves' Disease: Implications in an Endemically Iodine Deficient Area. Journal of Postgraduate Medicine, 47, 244-247. 